\title{
Anabases
}

ANABASES Traditions et réceptions de l'Antiquité

17 | 2013

Varia

\section{Le château de Bournazel, l'Antiquité retrouvée}

\section{Bruno Tollon}

\section{OpenEdition}

Journals

Édition électronique

URL : http://journals.openedition.org/anabases/4102

DOI : 10.4000/anabases.4102

ISSN : 2256-9421

\section{Éditeur}

E.R.A.S.M.E.

\section{Édition imprimée}

Date de publication : 1 mars 2013

Pagination : $51-68$

ISSN : 1774-4296

\section{Référence électronique}

Bruno Tollon, «Le château de Bournazel, l'Antiquité retrouvée », Anabases [En ligne], 17 | 2013, mis en ligne le 01 avril 2016, consulté le 21 octobre 2019. URL : http://journals.openedition.org/anabases/ 4102 : DOl : 10.4000/anabases.4102 
Anabases 17 (2013), p. 51-68.

\section{Le château de Bournazel, l'Antiquité retrouvée}

BRUNO TOLLON

LONGTEMPS LES VOIES DE LA CRÉATION, ou plus exactement leur étude, ont suivi les circuits définis clairement par la doxa du moment. À chacune de ses étapes, la Renaissance française empruntait à des modèles venus d'Italie et puisait dans un corpus apte à fournir aux artistes matière à copie simple, interprétation ou invention. Si l'on a beaucoup exagéré la portée des règles codifiées par les traités d'architecture, on a, en outre, utilisé une grille d'analyse identique pour les œuvres de la " France du Nord » et pour la production des cités méridionales : on n'a pas assez pris en compte le voisinage des monuments antiques que chacun pouvait longer, parcourir ou même visiter avec l'enthousiasme d'un découvreur de civilisation disparue ${ }^{1}$.

Le présent article a pour but d'examiner, à travers un groupe de sculptures ornant la cour du château de Bournazel, le contexte qui a permis de les voir naitre pour mieux en faire la lecture, à la manière des auteurs de livres d'emblèmes, qui articulent images et réflexions sentencieuses, et d'en tirer - faut-il le dire ? - la substantifique moelle.

Le château neuf, avec ses deux ailes fermant les côtés nord et est de la cour, est admirablement construit; depuis le XIX siècle, il a toujours retenu l'attention des voyageurs, des historiens et des dessinateurs attirés par les sites pittoresques ${ }^{2}$. Le nom du comman-

1 Pour le Moyen Âge on a été plus attentif à cette dette ; voir J. ADHÉMAR, Les influences antiques dans l'art du Moyen Âge français, Londres, Warburg Institute, 1938 (réimpression 1968) et V. Lasalle, L'influence antique dans l'art roman provençal, Paris, De Boccard, 1970 ; pour les récentes orientations, voir à ce propos le texte de présentation du colloque Le génie du lieu. La réception du langage classique en Europe (1540-1650), VI ${ }^{\mathrm{e}}$ rencontre d'architecture européenne, INHA, Centre André Chastel, 11-13 juin 2009.

2 La bibliographie la plus complète se trouve dans J. LOURGANT, «Images des monuments aveyronnais au XIX siècle et idéologie patrimoniale », Études aveyronnaises, 2008, p. 231-273. 
ditaire, Jean de Buisson, et la date de 1545, qui fixe l'époque des travaux de l'aile nord, sont les seules informations disponibles du fait de la disparition totale des archives familiales pendant la Révolution, ramenant l'enquête au seul examen de l'édifice lui-même et de ses sculptures. Or ces reliefs qui offrent un champ particulièrement complexe ont été, jusqu'ici, laissés dans l'ombre. L'étude de ce château, lors du Congrès de la Société Française d'Archéologie en Aveyron, a donné lieu à une première relecture du monument et a proposé une série de pistes pour la compréhension des sculptures de la cour ${ }^{3}$.

\section{La présence d'un dieu romain invite à revoir la lecture du monument}

L'identification des traits de Jupiter Amon sur la métope de la frise dorique, qui surmonte la porte d'entrée, nous a conduit à reconsidérer l'iconographie de l'ensemble des bas-reliefs qui la composent. Jusqu'ici seuls les protomes (avant-trains) de taureaux, mis en relation avec ceux du château d'Uzès, s'étaient vus tirés de l'oubli ${ }^{4}$. Les spécialistes de la civilisation romaine ont attiré notre attention sur cette métope et sa ressemblance avec les modèles augustéens, comme ceux mis en valeur au Musée des forums impériaux, dans les marchés de Trajan à Rome ; ou encore avec les clipei des portiques entourant les forums d'Auguste dans les grandes fondations d'Espagne, Tarragone ou Merida, et leur rôle (associés à la Méduse) d'indispensables éléments de la propagande impériale 5 .

3 Voir notre notice, "Le château de Bournazel ", Monuments de l'Aveyron, Congrès archéologique de France, SFA, Paris, 2009, p. 43-52.

4 F. LEMERLE, "Le bucrane dans la frise dorique à la Renaissance : un motif véronais ", Annali di Architecttura, 8 (1996), p. 85-92 et "L'entablement dorique du théâtre d'Arles et la diffusion du modèle dans l'architecture de la Renaissance ", Bulletin monumental, 1996, t. 154-4, p. 297-306. La chronologie proposée pour l'aile Renaissance du château d'Uzès a donné lieu à une mise au point de la part de B. Sournia et J.-L. VAYSSETTES, «La façade d'Uzès et son projet ", Monuments du Gard, Congrès archéologique de France, SFA, 1999 , p. 439-450.

5 D. CAZES, directeur honoraire du musée Saint-Raymond, musée des antiques de Toulouse, communication écrite (27 mars 2011) : "Sur le culte organisé à Rome et dans l'Empire à l'époque augustéenne "; G. SAURON, "Jupiter Amon dans le décor officiel des provinces occidentales ", in M. Navarro Caballos et J.-M. Roddaz (dir.), La transmission de l'idéologie impériale dans l'occident romain, Bordeaux-Paris, 2006, p. 205-220 et « Le forum et le théâtre : le décor du culte impérial d'Arles à Merida ", in Culto imperial y poder, Actes du colloque international, Merida, MNA Romano, Rome, 2007, p. 105-123. Il convient de rappeler que, depuis une génération au moins, l'attention des artistes était attirée sur ces monuments-là. Dans son Terzo Libro (1540), traitant des antiquités de Rome et d'Italie, Sebastiano Serlio exprimait déjà son intention de venir étudier les monuments romains de la Gaule (1540) et quelques années plus tard, par ordonnance datée de septembre 1548, le gouverneur du Languedoc, Anne de Montmorency, prescrivait des mesures pour 
Trois représentations retiennent d'abord l'attention : leur fidélité à un modèle précis les rend identifiables. La première figure se trouve au-dessus du perron d'entrée : bien qu'endommagée, elle conserve les éléments indispensables correspondant au dieu Jupiter Amon ; la divinité est vue de face. Ses traits nobles, sa chevelure abondante, sa barbe aux boucles soignées et les cornes dont il est doté, suffisent à l'identifier (fig. 1). Le même motif, intact cette fois, figure à nouveau à la troisième travée (fig. 2) et, plus significatif encore, au centre de la frise dorique qui surmonte la porte de la première pièce, dans le vestibule de l'aile nord (fig. 3). Les mêmes caractéristiques sont soigneusement reproduites et on y retrouve en particulier la coiffe, ou calathos, qui caractérisait le dieu égyptien. Sauf dans ce dernier cas, les autres effigies de Jupiter Amon sont associées à des chutes d'ornements : trophées d'armes ou guirlandes de feuilles et de fruits accrochés aux cornes de bélier, comme s'il était indispensable de souligner encore le caractère religieux et apotropaïque de la représentation.

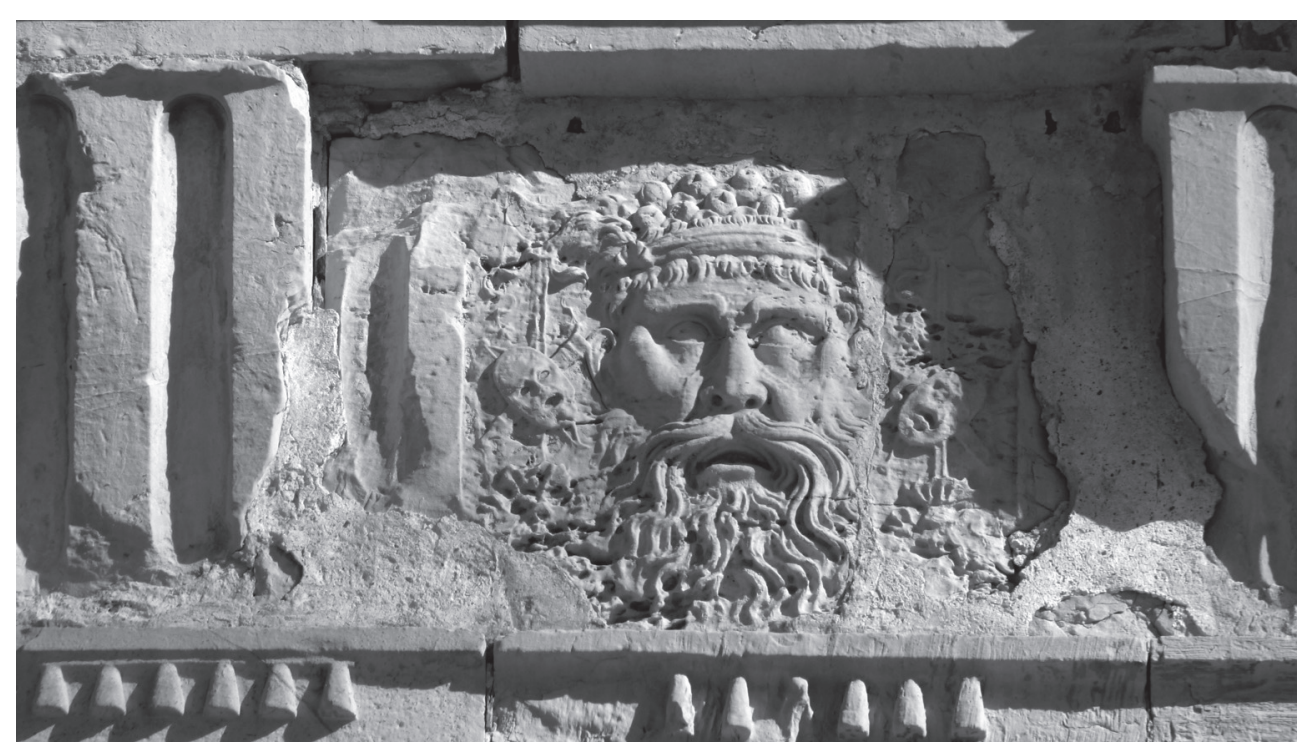

1. Château de Bournazel, métope de la frise dorique, au-dessus de la porte d'entrée de l'aile nord : Jupiter Amon, masque du dieu lié au culte impérial, chevelure et barbe soignées, doté des cornes de bélier et du calathos du dieu égyptien Amon.

la sauvegarde des antiquités de Nîmes dont les connaisseurs " tireront non seulement dilection mais encore beaucoup de profit pour l'art de l'architecture ". Un autre aspect de la question est abordé dans l'ouvrage de F. Lemerle, La Renaissance et les antiquités de la Gaule L'architecture gallo-romaine vue par les architectes, antiquaires et voyageurs des guerres d'Italie à la Fronde, Brepols, Turnhout, 2005. 


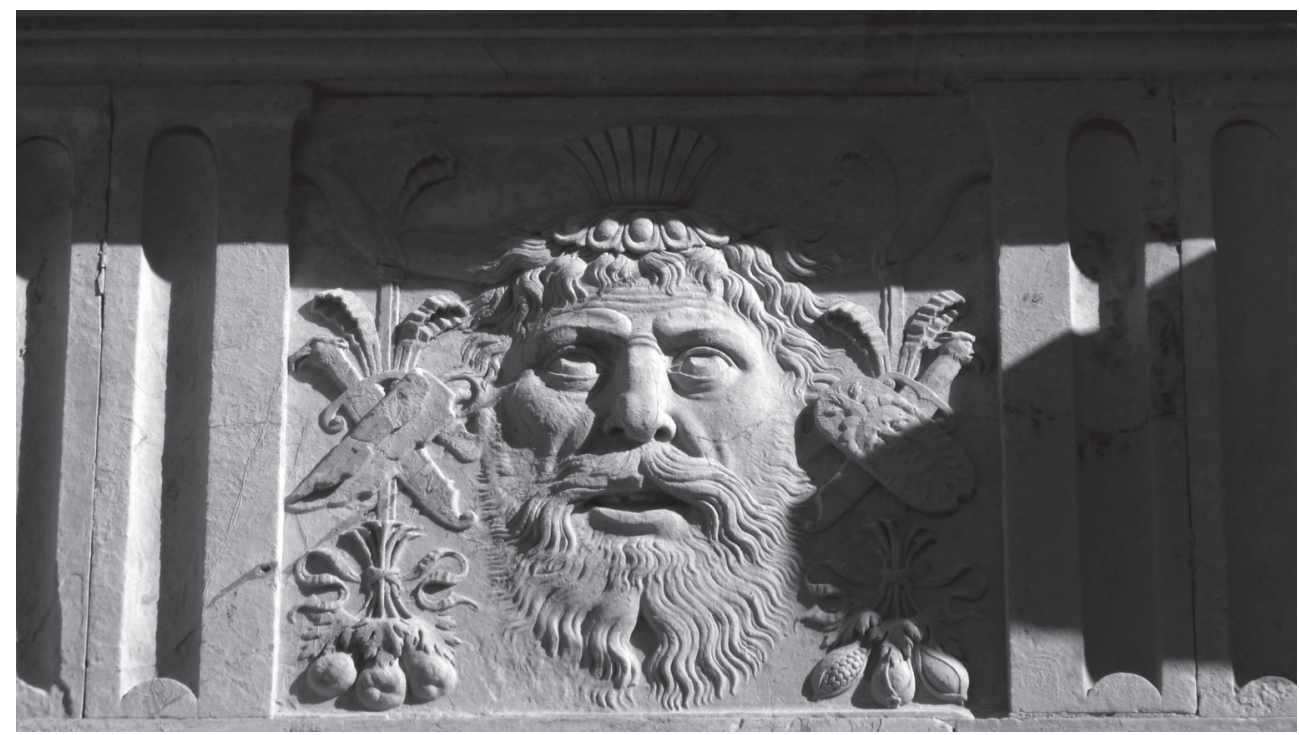

2. Aile nord, troisième travée, le masque de Jupiter Amon intact permet de juger des qualités plastiques de la sculpture.

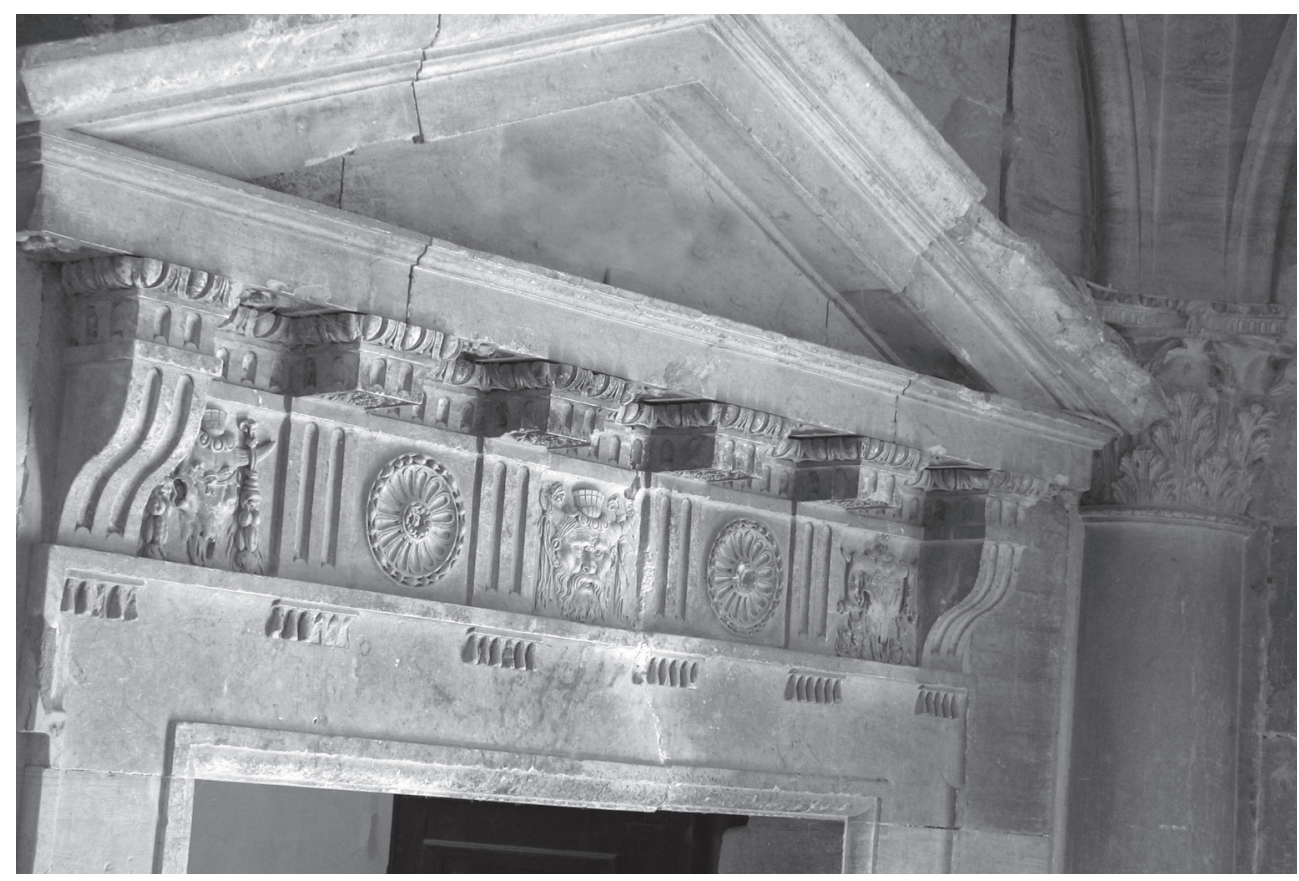

3. Vestibule d'entrée, aile nord, Jupiter Amon sur la métope centrale de la porte ouvrant sur la première pièce, avec calathos et bandelettes. 
Ces reliefs apportent la preuve que le sculpteur avait pu avoir sous les yeux des modèles de très grande qualité et en très bon état, mais, sans doute, déjà détachés de tout contexte architectural. Les villes de la Narbonnaise (Arles, Nîmes et Narbonne) n'ont pas conservé leur forum ; à Vienne (Isère) on peut voir encore un angle du portique romain où figure en place un relief proche d'un Jupiter Amon. En revanche des vestiges significatifs, retrouvés à Caderousse près d'Orange et à Narbonne, prouvent que des œuvres comparables pouvaient être accessibles aux amateurs de la Renaissance autant qu'aux sculpteurs appelés à des chantiers dans la région ${ }^{6}$. La présence de ces basreliefs témoigne du culte impérial dans les provinces de la Gaule romaine. La volonté de s'approprier les mérites et les bienfaits, le caractère propitiatoire de l'effigie ne fait pas de doute et invite à regarder plus attentivement les autres métopes sculptées sur la façade. Remarquons que le masque de la seconde travée est proche d'une Méduse, alors que ceux qui dérivent de Jupiter Amon déclinent toutes les possibilités du thème.

Le recours à des motifs romains précis et très exactement copiés renvoie à une réalité archéologique certainement familière, comme les bucranes, les têtes et protomes de taureau. Ces représentations sont enrichies d'autres motifs : trophées militaires (armes antiques et contemporaines, et même des "bâtons à feu ") ou chutes de fruits, accrochés aux cornes des animaux. Il faut y ajouter toutes les allusions aux campagnes militaires victorieuses qu'il s'agisse des combats antiques (ou fantastiques) avec héros antiques ou êtres hybrides : autant d'allusions à la carrière du commanditaire (fig. 4).

\section{L'iconographie des ressauts d'entablements}

Les dés cubiques (qui surmontent les colonnes de l'ordre dorique) ou ressauts d'entablement occupent l'emplacement d'une métope, mais leur iconographie reste indépendante de celle du reste de la frise. Ces cinq éléments cubiques ont reçu un décor

6 Les musées archéologiques conservent de beaux exemplaires, voir par exemple dans le Recueil général des sculptures sur pierre de la Gaule, sous la direction de H. LAVAGNE, Lyon, Académie des inscriptions et belles-lettres, Paris, 2006, n 107 et $\mathrm{n}^{\circ} 342$, des bas-reliefs de Jupiter Amon conservés au musée Saint-Pierre de Vienne ; au musée Calvet (Avignon), est conservé un médaillon hémisphérique, en marbre blanc, représentant Jupiter Amon, inv. G 155, provenant de Caderousse (Vaucluse) ; l'Annuaire de l'École pratique des hautes études, Section sciences historiques et philologiques, 21, 2005-2006, p. 111, mentionne la découverte d'un clipeus en marbre, orné de la tête de Jupiter Amon ; Jean Poldo d'Albenas, dans son ouvrage, admirablement illustré, Discours historial sur les antiquités [...] de la ville de Nîmes, Lyon, 1560, n'en cite aucun. Victor Lassalle, directeur honoraire du Musée archéologique de Nîmes, a bien voulu nous faire bénéficier de ses connaissances : il nous a signalé deux cas de l'influence antique, l'un appartenant à l'époque médiévale, un Jupiter Amon interprété sur un chapiteau roman de l'abbaye Saint-Ruf d'Avignon et l'autre, de la Renaissance, dans un hôtel particulier de Nîmes. Qu'il veuille bien trouver ici l'expression de notre gratitude. 


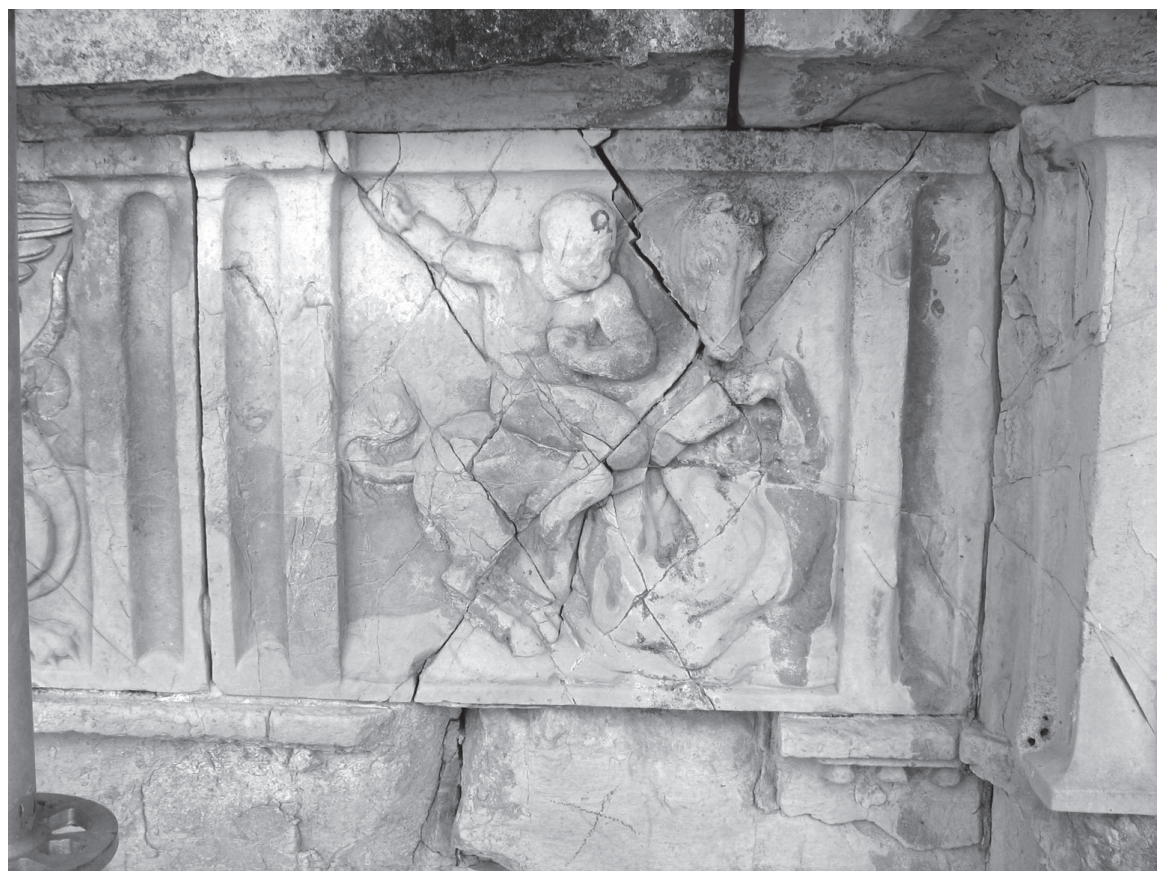

4. Aile orientale, métope, combat à l'antique entre cavalier et fantassin, inspiré par une plaquette de bronze « à l'antique».

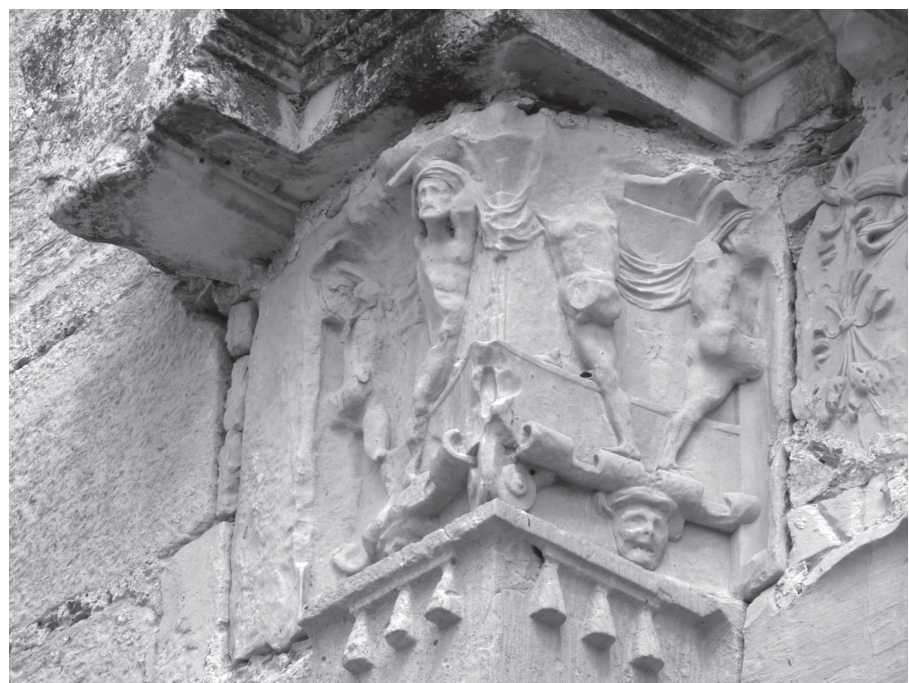

5. Château de Bournazel - Aile nord, $1^{\text {er }}$ ressaut de la frise dorique. Quatre héros accrochés à un (une) voile; la composition habile diversifie les attitudes et les raccourcis. 
réparti sur deux registres sauf pour ceux placés aux extrémités : le premier, à gauche, montre quatre hommes nus aux musculatures athlétiques (têtes et bras très endommagés) : les raccourcis habiles et l'enchaînement des postures de trois-quarts, comme le voile auquel ils semblent se retenir, suggèrent une scène unique pour une action toutefois impossible à préciser - sauf à découvrir le modèle antique, car le nu héroïque les renvoie à ce contexte (fig. 5). À l'autre bout de la façade, le dernier ressaut figure le dieu Pan assis de face, accompagné d'une faunesse. Ainsi un climat mythologique semble installé, que la présence de faunes et de sylvains, répandus sur les deux façades, vient confirmer pour aiguiller le spectateur vers un Âge d'or !

Les trois autres ressauts montrent un riche décor établi cette fois sur deux registres, comme sur les chapiteaux de la première Renaissance : ils ont en commun quelques motifs : les aigles, les putti, les masques bellifontains (mis à la mode par le chantier de Fontainebleau) qui ont tant divulgué le thème du satyre et du masque grimaçant. Cependant, dans chacun des trois ressauts, le centre de chacune des faces souligne un thème principal, bien mis en valeur par un médaillon, lui-même pris dans un cartouche en forme de « cuir ». De gauche à droite, on voit des nymphes dansant, séparées par des atlantes-faunes ; une écharpe mise en valeur dans une table ansée, répétée sur chacune des faces ; des masques féminins tirés du répertoire bellifontain. Si leur caractère emblématique ne semble pas faire de doute, du fait de leur emplacement, leur signification précise reste à définir.

Il n'en est pas de même pour le troisième ressaut qui condense à lui seul tous les thèmes du décor de Bournazel. Il montre l'organisation la plus complexe pour l'iconographie la plus riche. Sur le registre supérieur on trouve des aigles essorants et des Amours juchés sur des coquilles; le médaillon central est occupé par une figure féminine d'une séduisante nudité. Elle brandit un fanion (ou une voile) et pose la main gauche sur un bouclier timbré d'un gorgoneion. Autant d'éléments qui l'identifient comme une Venus Armata, la protectrice des hommes d'armes et du combattant vertueux (fig. 6). Dans une gravure d'Agostino Veneziano représentant la déesse, l'artiste vénitien lui conserve une armure et lui donne un Amour pour compagnon, alors qu'ici le putto de la coquille, placé au dessus, suffit à évoquer sa présence ${ }^{7}$. Les attributs retenus peuvent nous paraître incomplets; ils ne l'étaient pas à une époque où la représentation allégorique n'était soumise à aucune contrainte normative : deux attributs suffisent ici pour évoquer Venus Armata. Les aigles jupitériens confirment le caractère emblématique, comme les faunes des médaillons latéraux suggèrent l'Âge d'or d'une humanité soumise aux choix instinctifs (et éventuellement aux vices). L'observation attentive de la Vénus

7 Agostino dei Musi dit Veneziano (Venise ca 1490 - Rome 1540) publie en 1528 une gravure représentant Venus armata, nue et hanchée, une lance d'une main, un casque de l'autre; un Amour s'accroche à sa hanche et derrière elle est posé un bouclier à masque de Gorgone. Ce dernier est remplacé par une cuirasse et des putti armés de lances entourent la divinité, sur un dessin de Marco Zoppo, conservé au British Museum à Londres. Pour un thème en vogue les variantes ne manquent pas. 
permet de proposer une lecture plus complexe, mais vraisemblable en ces temps d'invention emblématique : le fait qu'elle se tient en équilibre sur le rouleau de la bordure du « cuir » découpé, invite à y voir la Fortune sur sa boule. On pourrait proposer alors une lecture jouant sur le double sens selon une dialectique propre à ce temps. Il est sûr, du moins, que la présence de Vénus s'inscrit dans le contexte plus général qui la met en rapport avec la Fortune dans une confrontation dialectique : la sentence cicéronienne Virtuti Fortuna Comes ("La Fortune favorise la Valeur») se justifie ici à propos d'un homme de guerre comme le propriétaire du château, soumis aux vicissitudes des combats et capable de force d'âme (virtù) dans les épreuves. Il s'identifierait alors avec un miles virtuosus ${ }^{8}$.

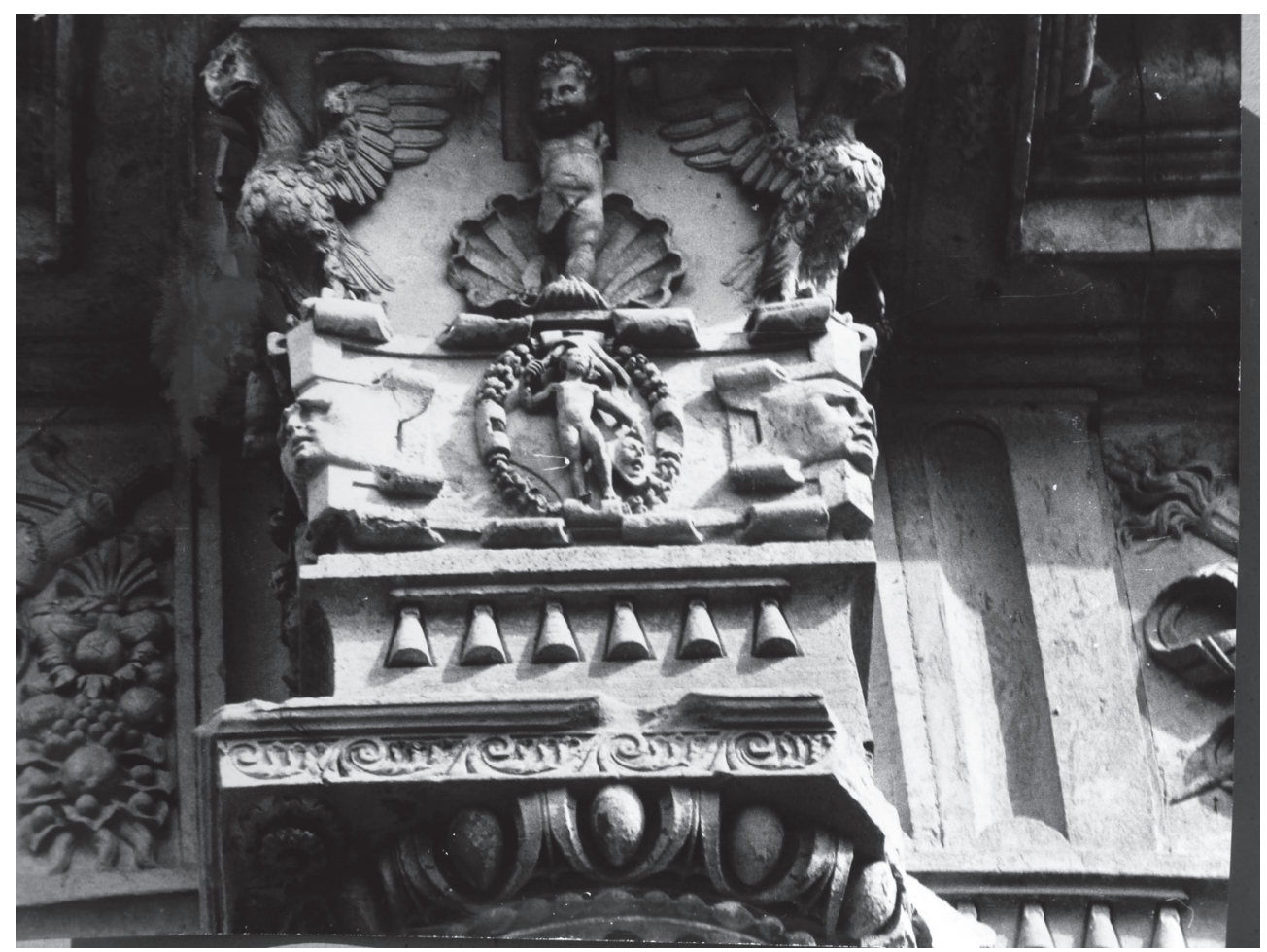

6. Aile nord, $3^{\mathrm{e}}$ travée, Venus armata, la jeune femme nue brandit un fanion (ou une voile) et s'appuie sur une targe, timbrée d'un gorgoneion.

8 Fortune, catalogue de l'exposition organisée par L. Galactéros de Boissier et Y. Giraud, Lausanne, 1984, p. 11-18 ; A.M. LECOQ, François Ier imaginaire : symbolique et politique à l'aube de la Renaissance française, Paris, Macula, 1987, p. 427, et sur le succès de l'iconographie renaissante de l'allégorie, F. ButTAY-Jutier, Fortuna: Usages politiques d'une allégorie morale à la Renaissance, Paris, 2008. 
L'hypothèse trouve en quelque sorte confirmation dans les deux métopes de la frise orientale de la cour, qui réitère de façon explicite cette fois en les juxtaposant les deux allégories complémentaires (fig. 7) : Venus Armata s'y retrouve confrontée à Fortuna placée à côté d'elle; toutes deux occupent la totalité de la métope, comme de véritables tableaux en plein air. On peut rapprocher ces représentations des reliefs du château d'Assier où Galiot de Genouillac (grand maître de l'artillerie de France) a répété, inlassablement, la devise qu'il a faite sienne ("J'aime Fortune ») et dont il a fait sculpter l'image dans l'escalier de l'aile d'entrée?.

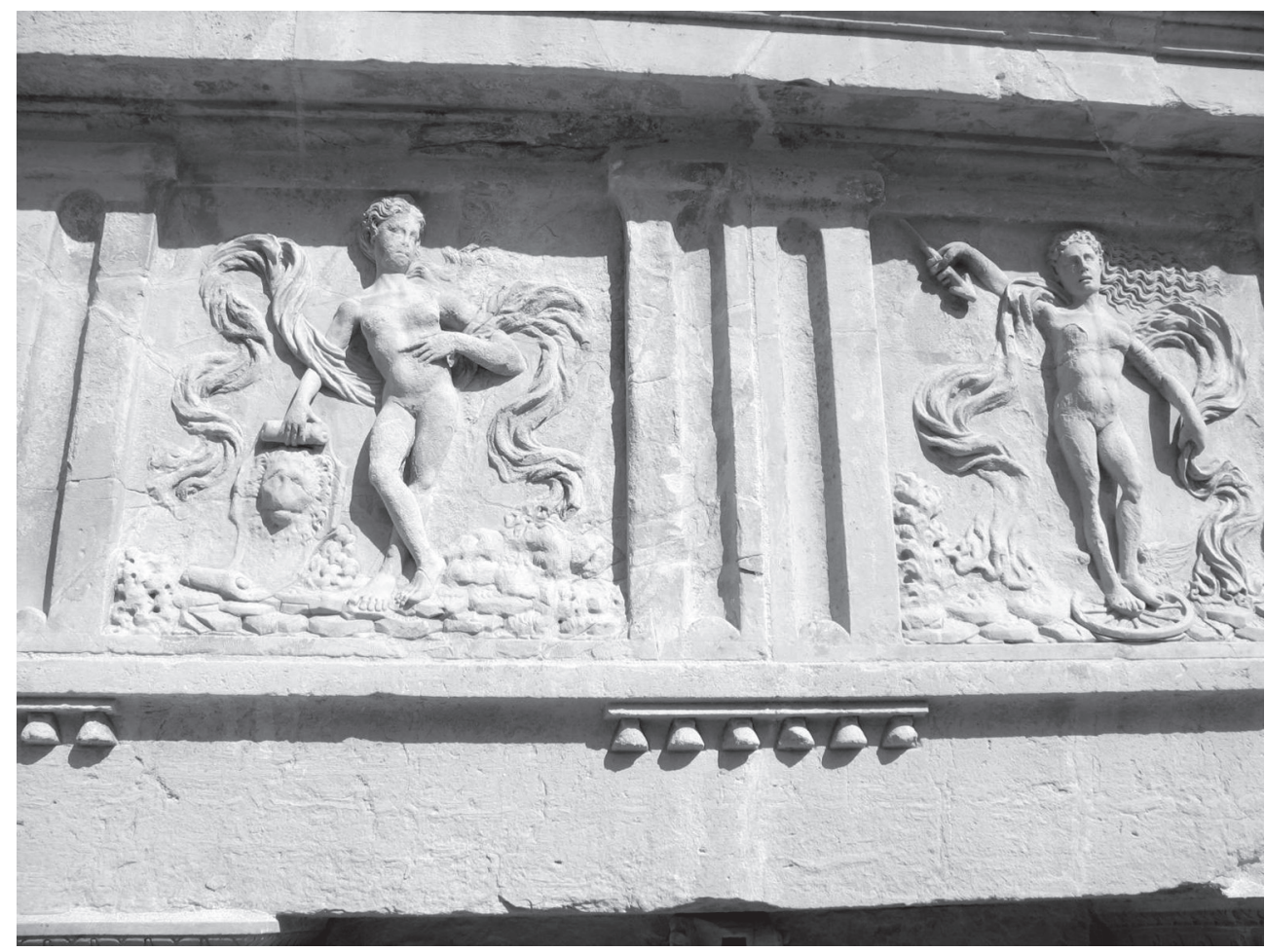

7. Aile orientale, $3^{\mathrm{e}}$ travée, deux métopes voisines, Venus armata et Fortuna: le sculpteur a associé les deux thèmes grâce à deux nus séduisants. L'iconographie ne déroute pas : de la main Vénus désigne la tête du lion, emblème du courage et de la force d'âme ou Virtù. À côté, Fortune réunit les attributs habituels : mer, roue, chevilles ailées, arme, cheveux au vent qu'il faut saisir.

9 F. Buttay-Jutier, Fortuna, p. 252-277 ; Bournazel semble, à plus d'un titre, avoir des thèmes communs avec Assier, comme nous le montrerons par la suite. 
La densité des figures et des motifs rassemblés sur les métopes ne suffisaient pas à satisfaire la fièvre emblématique du seigneur de Bournazel puisque tous les frontons, ceux des lucarnes comprises, ont été garnis non de simples médaillons ou figures d'ornement, mais de véritables bustes en ronde-bosse, au format réel comme s'ils étaient représentés « au naturel ». Bien individualisés par des attitudes pleines de vie, ils sont doués d'expressions destinées à les différencier les uns des autres. Ils different également par l'âge, le costume, et par les attributs permettant d'identifier la plupart d'entre eux. Sur les quinze personnages masculins on ne compte pas moins de quatre empereurs, avec des statuts et âges différents ${ }^{10}$. Et pour les autres (d'ailleurs confiés à d'autres praticiens moins doués), ils font partie du cortège de Preux popularisés par la littérature chevaleresque ${ }^{11}$.

Les cinq figures féminines, toutes aussi attachantes, peuvent illustrer la série parallèle des Femmes illustres ou Preuses, ces Belle Donne aussi populaires en France qu'en Italie. On remarque ainsi sur la troisième lucarne une Lucrèce et au premier étage, une héroïne, tête inclinée dont l'expression méditative ou douloureuse amène à s'interroger sur sa cuirasse dotée d'un col montant, renforcée par deux épaulières à l'effigie d'un lion (elle pourrait être assimilée à Zénobie, reine de Palmyre, vaincue par l'empereur Aurélien) (fig. 8). L'effigie la plus étonnante reste celle de la deuxième travée, avec sa chevelure en désordre dont les mèches sont devenues un grouillement de serpents, jusqu’à gagner ses épaules, où, malgré la partie sectionnée du corps des reptiles, deux têtes sifflantes viennent reposer. Est-ce vraiment la représentation de Méduse dont les masques s'installent sur les cuirasses - alors même qu'un gorgoneion est visible sur la chemise de l'héroïne (fig. 9) ? Jusqu'ici on ne la trouvait pas en rondebosse même si Victor Lassalle a attiré l'attention sur un modèle approchant dans un hôtel de Nîmes ${ }^{12}$.

Quelle que soit la véritable signification de la série, elle joue le rôle d'une galerie d'hommes et de femmes illustres, ces héros que le commanditaire semble avoir convoqués pour donner à la cour intérieure du château, par définition le lieu le plus idéologique de l'ensemble castral, un caractère solennel aux implications évidentes. Ces Hommes illustres ne sont réunis que pour s'associer au héros revenu des campagnes militaires et qui voudrait (sans doute "plein d'usage et raison"), profiter " du reste de son âge » et donner matière à réflexion en ayant recours à des moyens multiples mais bien choisis.

10 Nous tenons à remercier chaleureusement Daniel Cazes et Jean-Charles Balty, pour leurs observations et suggestions éclairantes.

11 On y reconnaît la main des auteurs des cariatides des cheminées et des figures engainées, aux fenêtres de la façade arrière de l'aile nord.

12 V. Lassalle a attiré notre attention sur un culot conservé dans la cour de l'hôtel de l'Académie de Nîmes, 16 rue Dorée (communication écrite, 11 septembre 2011) : cette tête de Méduse, sculptée, en très haut relief, semble directement inspirée d'un modèle antique. 


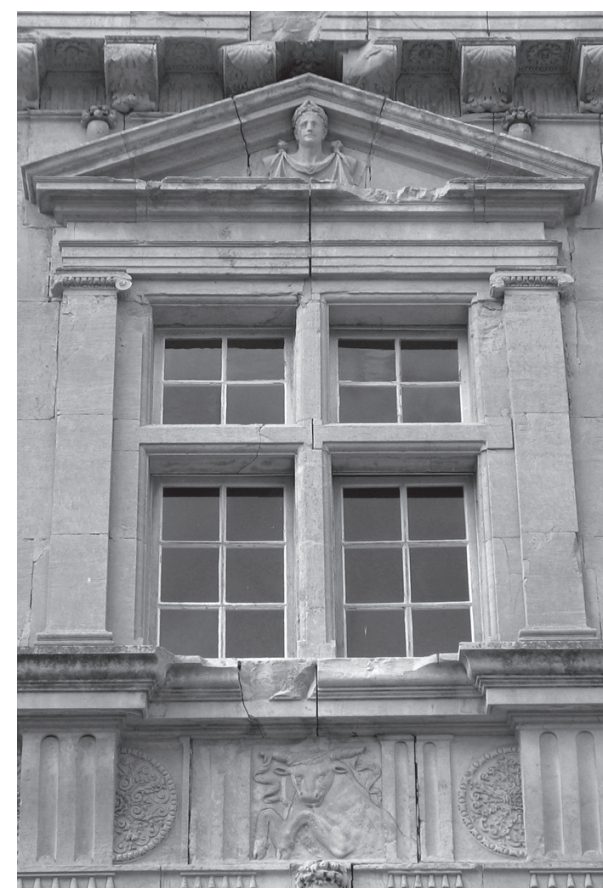

8. Aile nord, buste d'empereur romain lauré, de face et toge nouée sur les épaules.

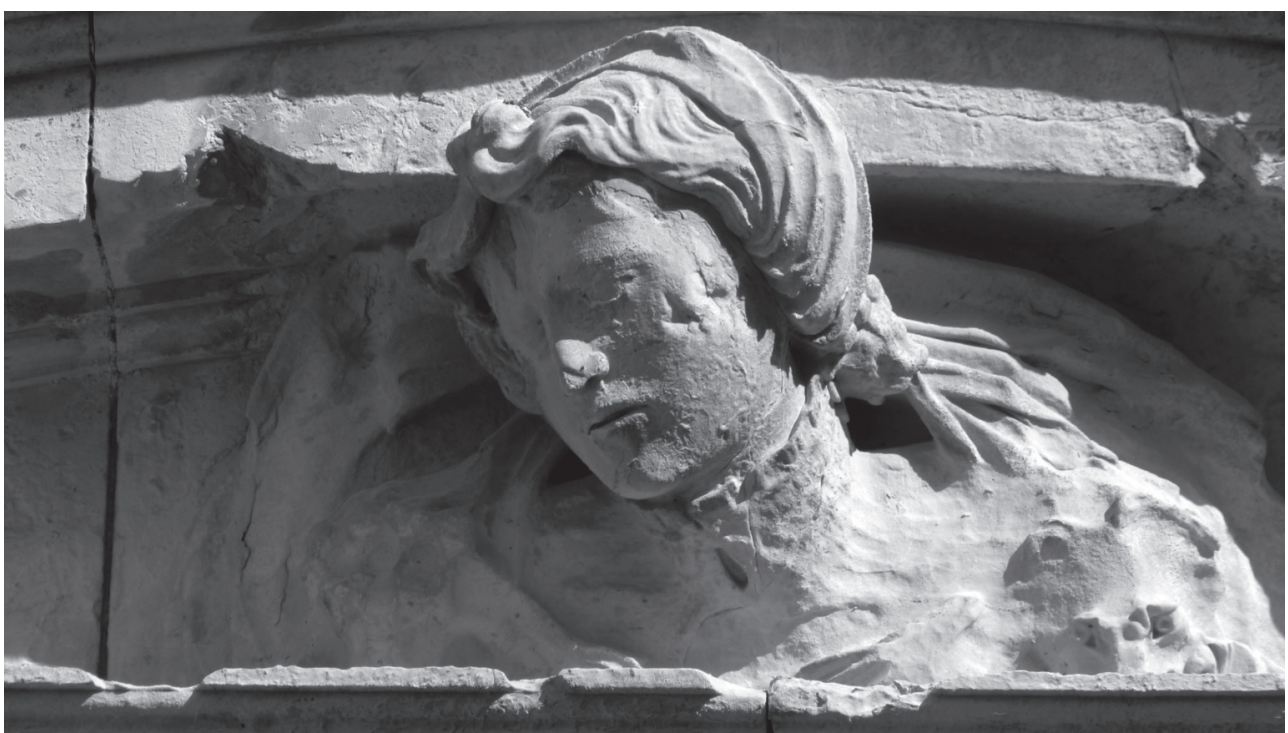

9. Aile nord, figure féminine penchée en avant, au visage méditatif : princesse guerrière, Zénobie ou la reine de Saba. 


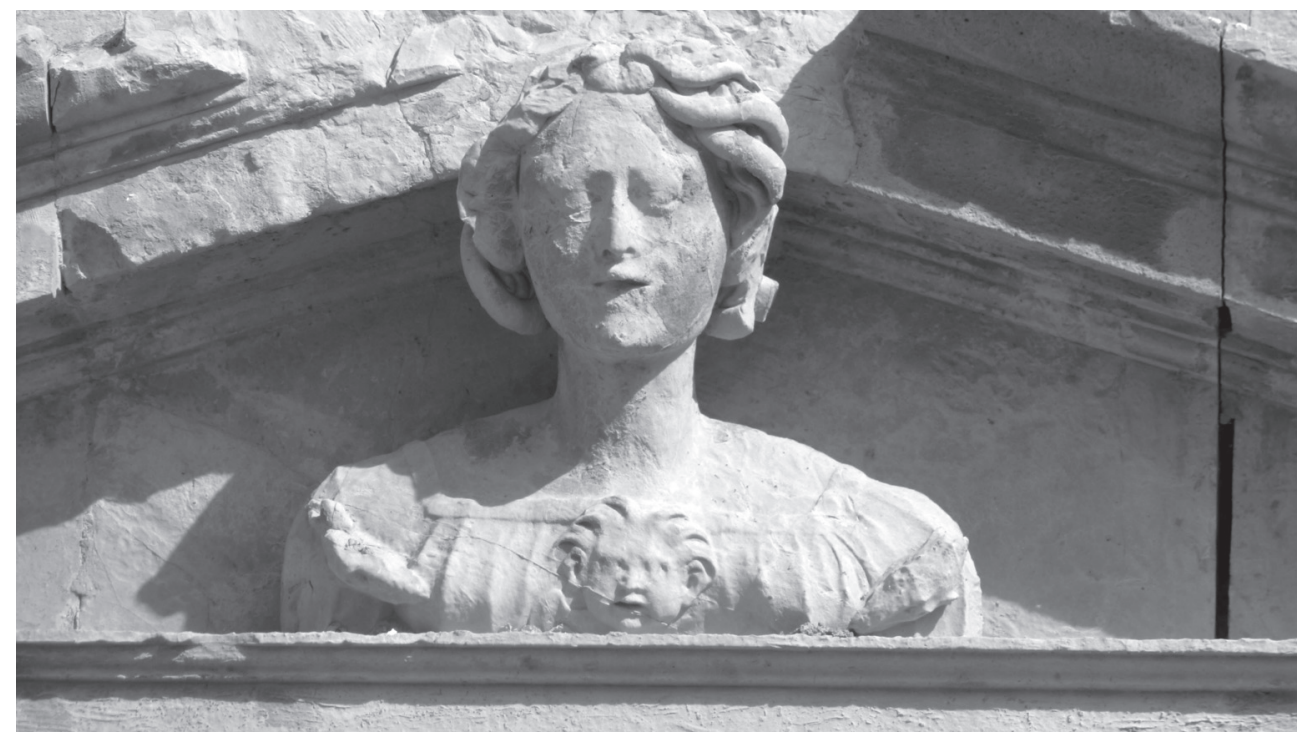

10. Aile nord, buste de Méduse aux têtes de reptiles posées sur les épaules.

Au total le sculpteur déroule, par métopes ou bustes interposés, une geste à la gloire du vertueux gentilhomme, faisant ainsi de cette suite une réplique de la fameuse litre sculptée ${ }^{13}$, commandée par Galiot de Genouillac, pour ceinturer l'église d'As$\operatorname{sier}^{14}$. La double référence à Fortuna et à Venus armata, retenue par Jean de Buisson, constructeur de Bournazel, répond à une époque où un gentilhomme, inconnu par ailleurs, peut s'approprier de telles déités, jusque-là retenues par les représentants des grands lignages. Audaces Fortuna juvat, l'époque assiste à la naissance d'une nouvelle catégorie d'hommes, les enfants de Fortune, ceux qui savent s'élever aux dignités et à la puissance par leur énergie et leur adresse, grands capitaines ou hommes d'action. C'est la leçon que véhiculent les emblèmes d'Alciat et à sa suite bien des auteurs, comme Guillaume de La Perrière à Toulouse ${ }^{15}$.

13 La litre sculptée remplace la frise peinte lors des cérémonies funéraires.

14 F. DE VAuX De Foletier, Galiot de Genouillac, Maître de l'artillerie de France (1465-1546), Paris, A. Picard, 1925, et B. Tollon, "Le château d'Assier » et "L'église d'Assier ", C.A.F., SFA, Quercy, p. 125-136 et p. 138-149 ; M.R. PRUnet-Tricaud, Le château d'Assier en Quercy, thèse de doctorat d'Histoire de l'Art de l'université de Paris-Sorbonne, 2003, 3 tomes (sous presse). Le château alignait, côté cour, des médaillons d'empereurs et d'autres bustes ornaient, semble-il, la galerie d'étage.

15 G. CAZAls, "La Perrière et l'humanisme civique ", in L'Humanisme à Toulouse (14801596), Actes du colloque international de Toulouse, mai 2004, réunis par N. DAuvoIs, p. 69-90. 


\section{À la recherche du commanditaire et de sa culture}

Qui est ce Jean de Buisson qui affirme ses vues par le truchement de l'emblématique ? Quelques trop rares repères : sa famille est originaire du Rouergue mais installée à Toulouse depuis près d'un siècle; il a reçu l'éducation propre à son milieu et sans doute comparable à celle dont bénéficièrent les héritiers des familles d'hommes d'affaires comme ceux de Jean de Bernuy, le célèbre marchand pastelier toulousain. Ces derniers deviennent magistrats ou hommes d'Église, avec des fonctions de premier plan. Il aurait pu en être de même pour le fils aîné du puissant banquier Hugues Buisson, seigneur de Mirabel en Rouergue ; or il choisit pourtant la carrière militaire comme son frère cadet. À la tête d'une compagnie de cinquante hommes d'armes, il a certainement participé à plusieurs des campagnes d'Italie et s'y est fait remarquer, puisque après la bataille victorieuse de Cérisoles (11 avril 1544), il obtient le collier de l'ordre de Saint-Michel ${ }^{16}$. Les capitaines d'ordonnance se rencontrent au cours des "montres " qui rassemblent les unités avant les campagnes militaires. C'est l'occasion de rencontres qui ne comptent pas moins que les liens noués lors des combats. Les unités formées en Rouergue, en Quercy comme en pays toulousain, soudent et élargissent ces solidarités de classe et d'origine ${ }^{17}$. Si l'on veut comprendre le personnage, dont on ne sait rien, et dans quel contexte ont pu s'établir les choix professionnels et culturels (dont cet abondant décor), il est indispensable de proposer un certain nombre de rapprochements pour tenter d'éclairer son horizon, son bagage intellectuel comme sa culture visuelle. La même démarche valant pour le sculpteur exécutant et sa culture artistique.

Le rapprochement entre certains éléments du château et de celui de Galiot de Genouillac à Assier, invite à donner à ce grand capitaine un rôle de modèle. Plus que la proximité géographique (Assier est à la limite du Quercy et du Rouergue), des intérêts communs lient d'ailleurs les deux familles à des occasions diverses ${ }^{18}$. Il en est de même,

16 L. D'Alauzier, "Les Boysson (Buisson), marchands à Aubin, Figeac et Toulouse ", in Rouergue et confins, Rodez, 1958, p. 109-119 ; A. NAVELlE, Familles nobles et notables de Toulouse et du Midi toulousain, 1995, t. II, p. 287-303 ; Baron DE GAUjaL, Études historiques sur le Rouergue, Paris, 1858, p. 141-142.

17 Sur l'organisation des compagnies et le rôle des solidarités régionales : N. Le Roux, La faveur du roi, Paris, Champ Vallon, 2000 et pour comprendre les réseaux de la noblesse provinciale et les profils de carrière de capitaine d'ordonnance, P.-J. SouRIAC, « Noblesse commingeoise et service armé du roi de France ", Revue de Comminges, 121 (2005), p. 523-550.

18 Quelques exemples de contacts avérés : un cousin de Buisson occupe la charge de viguier de Figeac, laissée à son profit par Galiot de Genouillac; les intérêts de ce dernier comptent dans le Rouergue méridional lié à Toulouse autant qu'au Quercy : son gendre, Charles de Crussol, lieutenant du gouverneur de Languedoc, fait son entrée dans Villefranche de Rouergue, siège de la sénéchaussée, le 27 février 1545 devant la noblesse venue de toute la province (BOUSQUET, Enquête sur les commodités du Rouergue en 1552, Toulouse, Privat, 1969, p. 117). 
sans doute, à propos du lieutenant général de l'artillerie, Charles de Crussol qui fut d'abord capitaine de cinquante lances, comme Buisson. Crussol, avait épousé la fille de Galiot, Jeanne de Genouillac, une personne de grande culture (dont la passion archéologique pour l'Antiquité était connue, en particulier de la reine de Navarre, la sœur de François $I^{\mathrm{er}}$ ). Elle a sans doute joué un rôle décisif dans les choix qui conduisent à la construction de l'aile nouvelle de son château d'Uzès. La date autrefois admise pour cette campagne de travaux été reportée aux années 1550. C'est plutôt sur l'inspiratrice des choix qu'il conviendrait de revenir. Car, à la mort de son père, l'héritier Antoine de Crussol n'a que seize ans et Jeanne de Genouillac, sa mère, qui dirige alors l'achèvement de l'église d'Assier, est en mesure d'assurer la direction des travaux ${ }^{19}$.

Le rapprochement entre les deux châteaux d'Uzès et de Bournazel se fonde sur le recours aux protomes de taureaux, inspirés par le théâtre d'Arles ${ }^{20}$; mais jusqu'ici, on n'a pas mis l'accent sur d'autres affinités qui les unissent avec le château de Marsillargues, construit par Jean de Louet de Nogaret ${ }^{21}$. Les trois châteaux ont en commun d'être entre les mains d'hommes d'armes à qui la carrière a apporté fortune et renommée. Ils peuvent construire une demeure selon leur rang. Crussol a la chance être l'époux d'une femme très cultivée et a sans doute adopté ses vues. En est-il de même dans les deux autres cas? Notons pour Bournazel la présence de bustes d'héroïnes, choisies avec soin.

Ces ensembles singuliers semblent marqués autant par le goût du moment, bellifontain pour le choix des cartouches, des pendentifs et des " compartiments ", que par l'adoption de motifs venus directement des monuments romains voisins ou découverts chez des amateurs "de choses rares et antiques ». Ces motifs rehaussent de leur prestige la résidence du gentilhomme soucieux de faire valoir sa bonne renommée. Un climat culturel de cet ordre a pu être à l'origine de l'étonnante création de Bournazel. L'entreprise a été longtemps interprétée à la lumière de la présence, à l'évêché de Rodez, du cercle qui gravite autour du cardinal d'Armagnac et de son secrétaire Guillaume Philandrier. D’autres pistes paraissent plus pertinentes : les rapports s'établissent plus directement avec Assier, autant pour des choix structuraux que décoratifs, et Montal, le château voisin construit aux mêmes dates par une cousine de Galiot, Jeanne de Balzac. Elle aussi adopta la Fortune (elle figure sur la porte d'entrée) ou les bustes, "au vif ${ }^{22}$ "

19 F. DE VAux de Foletier, Galiot de Genouillac, p. 100-105.

20 F. Lemerle, "Le bucrane dans la frise dorique à la Renaissance " ; B. Sournia et J.-L. VAYsSETTES, «La façade d'Uzès et son projet ».

21 B. Soumia et J.-L. Vayssettes, " Marsillargues ", dans Languedoc Roussillon. Le Guide du Patrimoine, dir. J.-M. Pérouse de Montclos, Hachette, Paris, 1996, p. 285-286.

22 C'est-à-dire représentant des personnages vivants, ce qui était inédit dans le cas d'un édifice privé et relevant de la noblesse de rang moyen. 
cette fois, de la châtelaine et de sa famille, célébrant son lignage, pour orner la cour ; mais aussi des allégories de la Force et de la Justice placées dans des niches ${ }^{23}$.

À Bournazel nous ne sommes plus dans l'apparat aristocratique de Galiot de Genouillac, troisième personnage de la cour, mais chez un gentilhomme de " moyen état ", pour reprendre la formule de Jacques Androuet du Cerceau à propos des catégories de châteaux ; les "gentilshommes des provinces ${ }^{24}$ " appartiennent, du moins ceux du Midi, à la nouvelle aristocratie urbaine. Leur réussite se concrétise le plus souvent par l'édification d'un château, car ils ont adopté tous les idéaux de la noblesse ancienne. On doit replacer dans un climat culturel de cet ordre l'étonnante création de Bournazel où se lisent l'ambition de parvenir et la nouvelle culture.

L'examen d'ensemble des façades d'Uzès et de Marsillargues conduit à pointer des similitudes du même ordre : toutes deux attestent de sources d'inspiration identiques. Elles ont en commun une dette vis-à-vis des antiques de Nîmes : les baies de l'une sont coiffées de frontons comme ceux du Temple de Diane ; les mêmes frontons caractérisent Marsillargues et mettent en valeur les tables décorées des trumeaux, devenues en quelque sorte des baies imaginaires. Ce champ décoratif mérite qu'on s'y arrête : il réunit, dans les deux cas, des motifs emblématiques très spécifiques dans des encadrements à crossettes et bordures en cuirs découpés, faisant intervenir des termes et des masques bellifontains. Ce cadre met en valeur un groupe de trophées d'une grande originalité à Uzès, où ils surmontent de petites scènes au dessin précis, consacrées à des combats de gladiateurs, certainement puisés à une source antique. Les fausses fenêtres de Marsillargues, encadrées de pilastres ioniques, célèbrent les combats mais par le biais d'un armet au riche cimier, entouré de chutes de fruits et banderoles et d'armoiries (bûchées) : Montal célèbre le lignage par le portrait, ici la noblesse se signale par l'emblème militaire le plus évident : le heaume de parade à visière fermée. Le rapport avec des modèles provenant des ruines antiques proches se vérifie sur l'attique, d'un format inhabituel : divisé en compartiments qui répètent le format inégal des travées, on y trouve de grandes guirlandes inspirées par des motifs funéraires, dérivés de l'Ara Pacis, et des dieux termes aux chevilles entravées, en guise de pilastres. Outre des bucranes, le motif le plus original revient aux têtes à l'antique : tête de feuilles dérivée d'un Jupiter Amon qui conserve encore les cornes du dieu égyptien; tête d'Attis avec son bonnet phrygien, tête de Bacchus et ses grappes de vigne (fig. 11). Une lecture détaillée devrait

23 S. Cueille et A. Dubin [Pascale Thibault], Le château de Montal. Itinéraire du Patrimoine, Paris, 2009, p. 12-14 et 32-49. On retrouve des bustes, des médaillons et des allégories des vertus dans un château encore méconnu à Usson en Charente-Maritime, qui présente plus d'une affinité avec Montal : F. GÉBELIN, Les châteaux de France, Paris, Les BeauxArts, 1927, p. 107 et 173 et J.-P. BABELON, Châteaux de France au siècle de la Renaissance, Flammarion-Picard, 1989, p. 253-255.

24 A. JounnNA, "La bonne noblesse des provinces ", et "valeurs nobiliaires ", dans La France de la Renaissance. Histoire et dictionnaire, Paris, Robert Laffont, 2001, p. 159-176. 
mettre en lumière l'intérêt de cet édifice encore méconnu, qui, par plus d'un trait, engage la comparaison autant avec Uzès qu'avec Bournazel ${ }^{25}$.

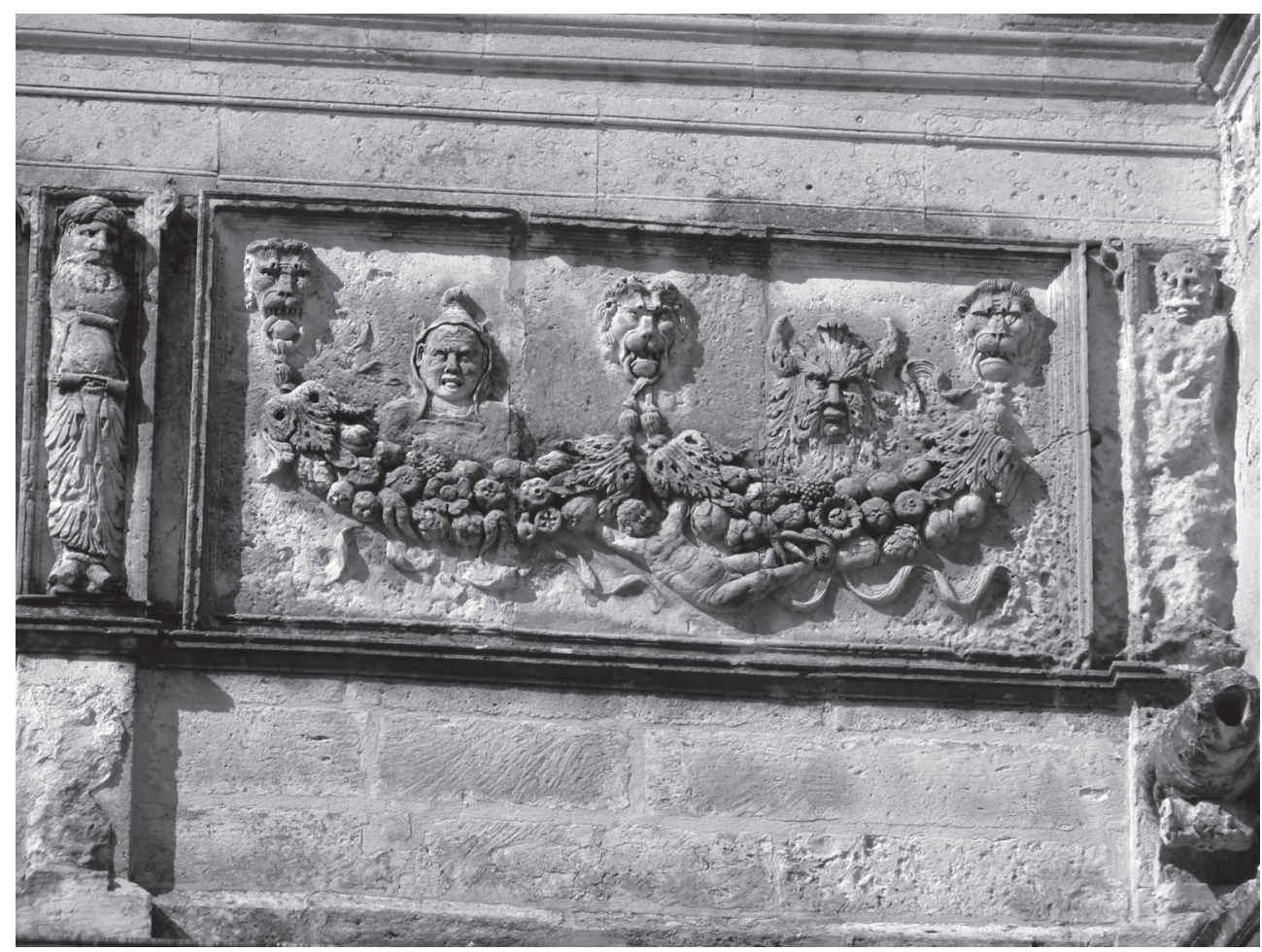

11. Marsillargues (Hérault), aile nord, façade sur cour, détail du décor de l'attique : volumineuse guirlande à l'antique où s'accroche le putto nu et deux masques à rapprocher de modèles antiques : un Attis au bonnet phrygien et un Jupiter Amon, que la chevelure et la moustache feuillagée transforment en tête de feuilles.

Jean de Buisson semble donc avoir voulu nous laisser un véritable portrait en creux, révélateur de ses ambitions comme de sa culture. Dans la cour d'honneur du château neuf, lieu idéologique s'il en est, se trouvent réunis les motifs propres à la célébration de son parcours, de la chance qui l'a accompagné et de sa réussite ; ce faisant, il témoigne aussi d'une série de choix révélateurs d'une nouvelle culture aristocratique. La puissance

25 Comme pour le château d'Uzès, à ce jour on ne dispose d'aucune analyse archéologique complète de la façade de Marsillargues ni de son décor, qui semble homogène et se rapporter par ses caractères stylistiques aux années 1550-1560. J.-L. VAYSSETTES nous a communiqué le dossier qu'il a réuni concernant la campagne de travaux pour l'aile en miroir de l'autre côté de la cour (1678-1679) ; qu'il en soit vivement remercié. 
seigneuriale s'exprime désormais moins par les « douves, pont-levis, créneaux et mâchicoulis $^{26}$ " que par ces nouvelles " marques " de noblesse, empruntées à l'Antiquité. Elles placent le seigneur et son château dans un contexte savant d'un nouveau genre, tiré des symboles de la religion romaine et des images triomphales de la propagande impériale ; ce jeu de références croisées complète l'apport d'allégories comme Venus Armata ou la Fortune. En se plaçant sous leur invocation, Jean de Buisson retrouve en quelque sorte Galiot de Genouillac et sa protectrice, et plus encore la pensée des humanistes, illustrée par le mot cicéronien, Virtuti Fortuna Comes, popularisé par la littérature emblématique ${ }^{27}$; le caractère séduisant de ces images nouvelles ne faisait pas de doute et on le vérifie en voyant comment Jean Imbert Dardenne, homme d'affaires avisé, installé à Toulouse mais originaire de Villefranche de Rouergue, s'en empare purement et simplement dans le petit château de Graves, construit dans le voisinage de cette ville. La réplique fidèle de la Venus Armata occupe le centre de la cheminée monumentale de la salle basse ${ }^{28}$.

La pluralité des modes d'expression symboliques et le caractère quasi jubilatoire des motifs et des personnages convoqués sont frappants. Une fois le thème installé, tout se passe comme si les allusions pouvaient se passer de rigueur et comme dans la pantomime, l'effigie de Jupiter Amon se métamorphoser et passer à la tête de feuilles ou au masque grimaçant, prisonnier d'un cuir bellifontain ; ou encore, pour la Méduse, à des variations plus éloignées encore de toute stricte répétition d'un modèle. Le décor remplit son rôle s'il apporte "bonne renommée ". La nouvelle culture élaborée dans les cercles d'amateurs et d'antiquaires a gagné cette nouvelle noblesse. Poldo d'Albenas à Nîmes et Guillaume de La Perrière à Toulouse sont des cas bien connus d'humanistes conseillers ; rappelons l'épisode de la visite de la reine de Navarre à Nîmes en 1536 : elle convoqua l'épouse du sénéchal Charles de Crussol ; on alla chercher à Uzès cette Jeanne de Genouillac, qu'elle avait connue à la cour et « il faisait beau voir ces deux grandes dames se promener ensemble parmi les monuments antiques et en discourir à plaisir comme les plus doctes ${ }^{29}$ ». On peut mesurer là un témoignage parfait de la nouvelle culture.

Un artiste est chargé de faire passer ces nouveaux messages. Les caractères de la production du maître de Bournazel le placent parmi les artistes qui s'inspirent aussi bien des formules ornementales inspirées par Fontainebleau que de l'antique. La circulation des modèles dessinés ou gravés est immédiate, comme Peter Fuhring l'a montré

26 P. Du Colombier, Le Château de France, Paris, Fayard, 1960, p. 33.

27 C. Balavoine, "L'emblématisation de Mercure à la Renaissance ", in Mercure à la Renaissance, Société française des seiziémistes, Poitiers, 1988, p. 64, à propos d'Alciat et de l'emblème CXIX de l'édition des Emblemata de 1531 ; A.-M. LECOQ, François Ir imaginaire, p. 427.

28 C. Corvisier, "Le château de Graves ", Monuments de l'Aveyron, C.A.F., SFA, Paris, 2011, p. $438-442$.

29 F. De Vaux de Foletier, Galiot de Genouillac, p. 127-129. 
à propos des cartouches de Du Cerceau, utilisés par le sculpteur au service de Geoffroy d'Estissac à Maillezay dès $1543^{30}$. À côté de ce langage, le maître de Bournazel possède une culture visuelle enrichie par les contactes répétés avec les monuments romains de l'ancienne Narbonnaise. Les modèles identifiés ici en apportent la preuve. Les ruines romaines d'Arles, de Nîmes ou de Narbonne fournissent un répertoire renouvelé, apte à séduire une nouvelle clientèle aux attentes différentes de celle du monde judiciaire ou des milieux intellectuels et humanistes et qui, dans le décor du château, peut se projeter avec ses rêves de chevalerie, de courtoisie et d'émotions.

Bruno TOLLON

Université de Toulouse (UTM)

Membre associé au Centre André Chastel, INHA, Paris

11, rue du Pic d'Aneto

31240 L'Union

btollon@yahoo.fr

30 P. Fuhrhing, "L'éuvre gravé », Jacques Androuet du Cerceau, J. Guillaume et P. Fuhrhing (dir.), Paris, 2010, p. 40-41, 305-306. 